\title{
Methylation and the human brain: towards a new discipline of imaging epigenetics
}

\author{
Corinde E. Wiers
}

Received: 12 August 2011/Accepted: 6 September 2011/Published online: 24 September 2011

(C) Springer-Verlag 2011

\begin{abstract}
The field of imaging genetics traditionally studies unidirectional associations between genes, brain functioning, and behavior. In a recent study by Ursini et al. (J Neurosci 31:6692-6698, 2011), imaging genetics methods are combined with epigenetic marks in living human beings. This approach may lead to a new field of imaging epigenetics, providing more mechanistic insight into causal pathways of how gene and environment interact and affect brain development.
\end{abstract}

Keywords DNA methylation - Epigenetics $\cdot$ Functional MRI · Neuroimaging · Gene-environment interaction · Cognition

Genes, brain activity, life experience, and cognitive functioning: how do these factors relate to each other? Over the last decade, association studies were considered to be the gold standard in the field of imaging genetics. These studies research the contribution of a variant of a specific gene to brain function, behavior, and mental illness [8]. An assumption in this line of research is that the influence of genes on brain and behavior is unidirectional and static over lifetime. In gene-environment interaction studies for instance, one tries to explain to what extent genetic makeup can contribute to individual responses to environmental

C. E. Wiers

Berlin School of Mind and Brain, Humboldt Universität zu

Berlin, 10099 Berlin, Germany

C. E. Wiers $(\square)$

Department of Psychiatry and Psychotherapy,

Charité-Universitätsmedizin Berlin, Campus Mitte,

Charitéplatz 1, 10117 Berlin, Germany

e-mail: corinde.wiers@charite.de influences (e.g., [2]). Genes, so the idea goes, let the brain develop in a particular way and determine your responses to the environment, and not vice versa.

There are however problems with this idea. Firstly, in the statistical analyzes of human association studies, genes and environment are often treated as separate factors. Separating gene and environment has been criticized as an assumption made more for historical than biological reasons [4], since genes can not be seen as operating in a vacuum free of the influence of experience. The statistical nature of these studies addresses how much variation in genotype accounts for variation in a trait, and the postulated biological implications are heavily based on the interpretations of researchers. So, despite their important explorative function, association studies do not provide mechanistic insights nor explanatory power at the level of the individual [6].

A second problem is that the assumption of a unidirectional relationship where genes code for RNA, are translated into proteins, determines brain development and in this way influence behavior, does not hold. Since the completion of the Human Genome Project, it has become clear that there are not enough nucleotides in human DNA to account for phenotypic variability. Further, molecular biological research has shown that epigenetic marks on the DNA influence the expression of these genes. Epigenetics literally means "above the genome" and accounts for gene changes that are not in the hard-wired DNA. DNA is wrapped around histone proteins, and the way these histones can be modified by mechanisms such as DNA methylation and acetylation, have influence on the accessibility of genes to be transcribed, while leaving the DNA code intact. These marks, as animal research reveals, can be changed by environmental influences such as diet, pharmaceuticals, and maternal care [6]. Nature and nurture 
are not separable anymore, but interact constantly throughout development.

Epigenetic processes can happen in the remodelling of DNA in post-mitotic, already differentiated cells, including neurons. Although the modifications are usually long lasting, in rare instances, they change over time and mark how much a gene is expressed at a specific time point. The more a gene is methylated, the lower the expression of the gene [9]. Most epigenetic studies into the human brain have been performed in postmortem brain tissue, and methylation patterns differ greatly over brain regions [5]. In studies with living humans, specific methylation patterns in blood have been related to psychiatric diagnoses, for example alcohol addiction [3] and change over time in twins [11]. The relationship between methylation status, brain activity, environmental experience, and behavior in living human beings as yet remains however largely unknown.

Recently, Ursini et al. [10] report that methylation of a single-nucleotide polymorphism (SNP) in the Catechol-Omethylatransferase (COMT) gene is not only associated with the degree of in vivo gene expression, but also with functional brain activity. The enzyme COMT breaks down dopamine (DA), and in humans, the gene contains a $\mathrm{G}$ to $\mathrm{A}$ polymorphism that translates into a methionine (Met) for valine (Val) substitution. The Val allele has an increased enzyme activity compared to Met, and is associated with responsiveness to stress, higher activation in the prefrontal cortex (PFC) during working memory (WM) tasks (i.e., reduced efficiency), as well as with psychiatric disorders such as schizophrenia [7, 8]. Importantly, Val creates a $\mathrm{CpG}$ dinucleotide, a site in the DNA string where methylation is possible, whereas the Met allele does not.

Ursini et al. [10] screened healthy participants on COMT genotype, and as expected, since methylation can occur in the Val allele only, the degree of methylation (measured in peripheral blood cells) in Val homozygotes was higher than in heterozygotes. Interestingly, although more lifetime stress was associated with reduced methylation in the $\mathrm{Val} / \mathrm{Val}$ subjects, this effect was not present in heterozygotes. Reduced methylation in the Val homozygotes was related to an increase in COMT mRNA expression, as well as positively correlated with WM performance. Additionally, the interaction of methylation and stress modulated brain activity during WM: the greater the stress and the lower the methylation, the higher the PFC BOLD response during the WM task. The authors interpret this as a reduced cortical efficiency, due to stress and methylation of COMT.

This imaging genetics study shows for the first time that gene methylation and environmental stress may modulate brain functioning and cognition in humans in vivo. It combines methylation in blood with fMRI, lifetime stress assessment, and mRNA expression of the gene in blood cells. This novel approach may serve as an example for a new field of imaging epigenetics, relating epigenetic mechanisms to neurocognitive function. Compared to imaging genetics, imaging epigenetics can provide insight into a more system-based approach of gene and environment interactions and is open to the possibility of environmental influences changing DNA expression, rather than seeing the genome as static.

Despite the strengths of this approach, the nature of the study is correlational and does not provide causal insight into the measured factors. Future studies should focus on causal relationships between brain function and epigenetic folding. For example, when methylation patterns change due to chromatin-modifying drugs, such as histone deacetylase (HDAC) inhibitors, does this directly influence brain functioning and behavior? Furthermore, it is still unclear how methylation in brain and blood relate to another. Ursini et al. [10] compare brain and blood from rats and argue that the finding of a similar methylated COMT region in rats will also be found in humans. However, since the COMT Val158 SNP is present in humans only, this argument seems to be weak. As suggested before [1], more comparisons between epigenetic marks in peripheral tissue and (postmortem) brain tissue are hence needed.

In conclusion, although the combination of epigenetic research and behavioral neuroscience is still very young, there is no doubt that more studies in this field will follow. By accepting the dynamics of the genome, it will however remain a challenge to integrate the methods of genomics, epigenomics, and neuroimaging so that meaningful conclusions can be drawn.

Acknowledgments Many thanks to Henrik Walter, Thomas Eastcott, Georgina Torbet and David Linden for their comments, and to Felix Bermpohl and Henrik Walter for their support.

\section{References}

1. Bogdan R, Hariri A (2011) "Understanding how life experiences influence biology is one of the major questions of modern science..." Evaluation of: [Ursini G et al (2011) Stress-related methylation of the catechol-O-methyltransferase Val158 allele predicts human prefrontal cognition and activity. J Neurosci 31(18):6692-6698. doi:10.1523/JNEUROSCI.6631-10.2011]

2. Caspi A, Sugden K, Moffitt TE, Taylor A, Craig IW, Harrington H, McClay J, Mill J, Martin J, Braithwaite A, Poulton R (2003) Influence of life stress on depression: moderation by a polymorphism in the 5-HTT gene. Science 301:386-389

3. Heberlein A, Muschler M, Frieling H, Behr M, Eberlein C, Wilhelm J, Gröschl M, Kornhuber J, Bleich S, Hillemacher T (2011) Epigenetic down regulation of nerve growth factor during alcohol withdrawal. Addict Biol. doi: 10.1111/j.1369-1600.2010. 00307.x. [Epub ahead of print]

4. Keller EF (2010) The mirage of a space between nature and nurture. Duke University Press, Durham \& London

5. Ladd-Acosta C, Pevsner J, Sabunciyan S, Yolken RH, Webster MJ, Dinkins T, Callinan PA, Fan JB, Potash JB, Feinberg AP 
(2007) DNA methylation signatures within the human brain. Am J Hum Genet 81:1304-1315

6. Meaney MJ (2010) Epigenetics and the biological definition of gene $x$ environment interactions. Child Dev 81:41-79

7. Meyer-Lindenberg A, Weinberger DR (2006) Intermediate phenotypes and genetic mechanisms of psychiatric disorders. Nat Rev Neurosci 7:818-827

8. Mier D, Kirsch P, Meyer-Lindenberg A (2010) Neural substrates of pleiotropic action of genetic variation in COMT: a metaanalysis. Mol Psychiatry 9:918-927

9. Tsankova N, Renthal W, Kumar A, Nestler EJ (2007) Epigenetic regulation in psychiatric disorders. Nat Rev Neurosci 8:355-367
10. Ursini G, Bollati V, Fazio L, Porcelli A, Iacovelli L, Catalani A, Sinibaldi L, Gelao B, Romano R, Rampino A, Taurisano P, Mancini M, Di Giorgio A, Popolizio T, Baccarelli A, De Blasi A, Blasi G, Bertolino A (2011) Stress-related methylation of the catechol-O-methyltransferase Val158 allele predicts human prefrontal cognition and activity. J Neurosci 31:6692-6698

11. Wong CC, Caspi A, Williams B, Craig IW, Houts R, Ambler A, Moffitt TE, Mill J (2010) A longitudinal study of epigenetic variation in twins. Epigenetics 5:516-526 